"light," using the term in its technical sense. The good results in some cases are not in dispute. These benefits can, however, be obtained in general by $X$ rays, and the advantages of the tungsten arc remain to be proved. It is claimed that there can be no ill-effects-a bold claim, surely, to make so soon, when the history of the Roentgen ray is romembered. In the treatment of any lesion when a deep effect is sought, it would seem foolish to use a feebly penetrating radiation. Anyone who has had experience of the rapid and remarkable effect of $X$ rays on Graves's disease will not be likely to abandon them for any new method without some very strong reason.

It would be much better to speak of the "tungsten arc" than of the Simpson light-or, at any rate, of the "Simpson tungsten arc"; this would tend to take away the air of mystery which seems to be gathering round the subject. As the rays in question are on the whole rather more penetrating than those from other sources of ultra-violet light they should certainly be investigated therapeutically. Some cases may be found which do better under them than under $\mathrm{X}$ rays; but any suggestion that a new therapeutic agent has been discovered is strongly to be deprecated. I have heard of advanced cases of deep-seated cancer being treated by the "Simpson" light. The rays probably stimulate general metabolism, as will any form of light; but only the very ignorant could suppose them capable of reaching and directly affecting an internal growth.

I am, Sir, yours faithfully,

Cavendish-square, W., March 4th, 1916 .

F. HERNAMAN-JOHNSON.

\section{DIAGNOSIS OF RUPTURED INTESTINE.}

To the Editor of THE LANCET.

SIR,-Whilst congratulating Mr. R. P. Rowlands on the well-deserved success which he obtained in the treatment of his case of ruptured intestine ${ }^{1}$ I should like to add somewhat to his remarks on the diagnosis of these lesions. Surgeons since the time of John Croft, if not before, have placed the greatest reliance on the symptoms which $\mathrm{Mr}$. Rowlands has accepted as the most importantpain, tenderness, and rigidity of the abdominal muscles. Having considered the subject of abdominal injuries for some years, and had some experience as a hospital surgeon, I have come to the following conclusions as regards rupture of the small intestine. ${ }^{2}$ They may be divided into three groups.

(a) Those in which there is shock, vomiting, acute abdominal pain, with great tenderness over the part struck, and board-like rigidity of the abdominal wall. All these symptoms are present, but they vary somewhat in their intensity; at one time shock is the main symptom, at another it is the pain, and so on. With these symptoms will be found a certain amount of dullness on percussion. This group forms about 50 per cent. of all cases.

(b) In this group there is no evident shock, and perhaps the patient goes to the hospital on foot or goes home, congratulating himself that he has had * "narrow escape"! He may have vomited after the accident which made him feel faint, but there are no marks of injury on the abdominal wall or they are but slight. He has considerable local pain and there is rigidity of muscle, sometimes confined to the side of the abdomen which was struck. There

1 The Lancet, Feb. 26r,h, 1916, p. 459.

2 The Acute Abdomen, p. 39, second edition. is tenderness on pressure and perhaps localised dullness, but the man feels that he will soon get over it, and probably remains under treatment with reluctance, or neglects to call in medical advice when he gets home. This group forms about 35 per cent. of the total number of cases.

(c) In the third group the symptoms are more indefinite; there is a history of abdominal injury, probably of the kind which sometimes produces a rupture of the intestine, but the shock is trifling; there is no vomiting, local pain is slight or absent, there is little tenderness, no rigidity of muscle, whilst percussion shows no change. After a variable time there may be a rising pulse, with that change in facial aspect which indicates to the experienced eye the presence of grave peritoneal inflammation. It may not be easy to say at what moment this commenced, but "a change has taken place." It may develop after an attack of vomiting, as in the case under the care of the late Mr. Walsham. That surgeon, who was patiently watching for symptoms in a case of this kind, found a complete change after the patient had vomited, and operating at once, gained a success. In yet another patient the onset of serious symptoms may be sudden and unexpected, caused by the giving way of a portion of severely contused bowel.

In the large majority of recorded cases the presence or absence of dullness on percussion is not mentioned in the published account, and an extensive extravasation of blood due to ruptured mesentery is very rare. A localised dullness, caused by a limited hæmorrhage, escape of intestinal fluid, or collapsed gut is not unusual.

The St. Thomas's statistics of the results of operation for the years 1886 to 1910 inclusive were 31 operations with 10 recoveries, whilst during the same period seven others were admitted which died from the injury. They were mostly too far gone to permit of operation. The small intestine was ruptured in 25 (of these five recovered), the large intestine in six, of which five recovered.

My late colleague, Mr. Bernard Pitts, used to advise that exploration should be done where there was a clear history of abdominal injury, such as by the kick of a horse or a mule, no matter what symptoms were present when the surgeon saw the patient. Few surgeons will disagree with this advice. I am, Sir, yours faithfully,

Harley-street, W., Feb. 25th, 1916. W. H. BATTLE.

\section{INOCULATION OF CIVILIANS AGAINST THE ENTERIC GROUP. \\ To the Editor of THE LANCET.}

SIR,-The far-seeing annotation in THE LANCET of Feb. 12th, and Dr. R. Donaldson's suggestions in the issue of Feb. 19th, concerning the recent outbreak at Reading, deal with public and individual prophylaxis against the infection, including the isolation of the carriers as well as of the sufferers. A further duty is to deal with its supply by suppressing the carrying evil. This would not be suppressed by merely curing our carriers if we disposed of a cure for them-for want of any being put into operation we are still wasting much money, medical labour, and military service in isolation hospitals. There is no other way but "to prevent its possibility" by an efficient bowel sanitation as part of the treatment of the fever. For the febrile, as well as for the cured typhoid carrier (and also for the typhoid-intact or contact 